\title{
La gestión de los centros de educación parvularia en la región del Maule (Chile): visiones desde la práctica educativa*
}

\author{
Nibaldo Benavides-Moreno a \\ Sebastián Donoso-Díaz ${ }^{b}$ \\ Daniel Reyes Araya ${ }^{c}$ \\ Tomás Neira Iturrieta ${ }^{d}$
}

\section{Resumen}

Desde hace más de dos décadas Chile ha impulsado iniciativas importantes de mayor cobertura y calidad de la educación parvularia, las que requieren -para su sustentabilidad- reforzar los procesos de gestión de los centros educativos, problemática de la que no se posee antecedentes sistemáticos. Atendiendo esta situación, se busca precisar aquellos obstáculos que directoras y educadoras de párvulos estiman como las más importantes, con la finalidad de proponer políticas para su mejoramiento. En este contexto, se desarrolló el estudio (exploratorio-cualitativo), a partir de un cuestionario de respuesta abierta. Los resultados identifican problemas claves que demandan pronta atención, a fin de contribuir al mayor impacto de las iniciativas señaladas.

Palabras claves: Educación parvularia. Gestión educacional. Política educacional. Sustentabilidad. Chile

\section{Presentación}

Chile, siguiendo la tendencia internacional y la evidencia respecto a la influencia e impacto de la educación parvularia, ha invertido en forma sustantiva en

\footnotetext{
* Estudio apoyado financieramente por el fondo UTALCA - IIDE 2017-06 y también por el Centro de Desarrollo del Liderazgo Educativo - CEDLE (Chile), Proyecto 12.

a Instituto de investigación y Desarrollo Educacional. Universidad de Talca, Talca, Chile

b Instituto de investigación y Desarrollo Educacional. Universidad de Talca, Talca, Chile

c Instituto de investigación y Desarrollo Educacional. Universidad de Talca, Talca, Chile

d Centro de Estudios del Ministerio de Educación. Santiago, Chile
} 
ella en las últimas décadas (ORGANIZACIÓN PARA LA COOPERACIÓN Y EL DESARROLLO ECONÓMICO, 2017). No obstante los avances registrados en cobertura y calidad de la enseñanza del nivel, resulta relevante para capitalizarles contar con sólidas propuestas de gestión para los centros educativos, avanzándose a la fecha de manera parcial en algunas dimensiones en este plano, a saber:

Elaborar marcos para la buena dirección y la buena enseñanza en jardines infantiles fortaleciendo el rol de directoras y educadoras de párvulos. Profesionalizar el rol de directoras en las salas cuna y jardines infantiles. Guiar y explicitar la generación e implementación de buenas prácticas en los procesos de enseñanza y aprendizaje en los primeros años. El marco referencial para ello estará vigente a contar del 2017 (CHILE, 2016, p. 30).

La Organización para la Cooperación y el Desarrollo Económicos (OCDE) sugiere que para atender deficiencias de calidad y equidad del sistema parvulario, Chile debe esforzarse en registrar sus prácticas exitosas al respecto (ORGANIZACIÓN PARA LA COOPERACIÓN Y EL DESARROLLO ECONÓMICO, 2017, p. 85). Asumiendo la diversidad institucional en este ámbito (Municipios, Junta Nacional de Jardines Infantiles -JUNJI-, Fundación INTEGRA, Servicios Locales de Educación -SLE-, e instituciones privadas), la JUNJI ha implementado en forma gradual un Modelo de Gestión de la Calidad de la Educación Parvularia, basado en la definición de estándares de gestión "orientado(s) a generar y estimular el mejoramiento continuo de los procesos educativos" (JUNTA NACIONAL DE JARDINES INFANTILES, 2013a, p. 3).

El artículo analiza las opiniones de directoras y educadoras de jardines infantiles de educación pública de la región del Maule (Chile), respecto a algunas dimensiones claves de sus prácticas de gestión, con la finalidad de (i) identificar las principales dificultades de gestión de los centros educativos; (ii) precisar los desafíos sustantivos que requieren soporte, y (a partir de todo ello) (iii) formular orientaciones de políticas para fortalecer este sector estratégico del desarrollo educativo del país.

La región en estudio es un territorio que concentra el 7\% de la población escolar del país, con alta vulnerabilidad, la que asiste masivamente al sistema público de educación, y con mayor razón en el nivel parvulario (INSTITUTO NACIONAL DE ESTADÍSTICAS, 2018). 


\section{La Educación Parvularia Chilena}

La educación y cuidado de niños menores de 6 años ha sido un tema prioritario en los programas de gobierno de las últimas dos décadas. En los años 90 los gobiernos democráticos promovieron una reforma sustantiva del nivel parvulario, cuyo eje fue la expansión de la cobertura y de su calidad. En 1997, el Ministerio de Educación impulsó el Programa de Mejoramiento de la Calidad y Equidad de la Educación (MECE), que destina el 16\% de sus recursos a la educación parvularia (CHILE, 2001a).

Estas medidas, junto a la integración del componente preescolar como parte de la estructura institucional del Ministerio de Educación, y la creación (1995) del Sistema de Evaluación Integrado para Párvulos (EVALÚA), impactaron positivamente en sus objetivos centrales: aumentar la cobertura y mejorar la calidad y equidad de la educación parvularia (BLANCO, 2012). En lo primero se avanzó del 20\% en 1990 al 30\% en el 2000, pero con un claro sesgo socioeconómico (HERRERA; BELLEI, 2002), mientras que respecto a lo segundo, el desafío es mayor dado que concurren factores como el apoyo de la familia, el trabajo de la educadora, la calidad de las interacciones educativas, los materiales y contextos de aprendizaje (CHILE, 2001b).

En el año 1999 se integra oficialmente a la Educación Parvularia como el primer nivel del sistema educativo, adquiriendo carácter obligatorio el segundo nivel de transición (5 a 6 años de edad). A comienzos del 2000, el gobierno amplió la cobertura de la educación parvularia, “... entre el 2001 y 2006 se incorporaron 120.000 niños y niñas al sistema, en más de 800 jardines infantiles públicos" (ADLERSTEIN, 2012,p. 38). Al 2010 se habían sumado más de 85 mil niños de los dos quintiles más pobres, aumentando de 781 a 4.300 los jardines infantiles, salas cuna y niveles parvularios.

El subsistema actualmente está organizado en dos ciclos: el primero de 0 hasta 4.0 años de edad, y el segundo de 4.1 a 6 años. El primer ciclo se divide en los subniveles, Sala Cuna ( 0 a 2.0 años) y Nivel Medio (2,1 a 4,0 años), mientras que el segundo ciclo se fragmenta en Primer Nivel de Transición (4,1 a 5,0 años) y Segundo Nivel de Transición (5,1 a 6,0 años). Al año 2016 había 4.229 instituciones de educación parvularia no ligadas a centros escolares; de ellas, 3.132 (74\%) son establecimientos JUNJI y 1.097 (26\%) de la Fundación INTEGRA, los que atienden a 259.158 niños(as). Si se suman los establecimientos educaciones de educación regular que ofrecen educación parvularia, son 10.318 instituciones y 785.368 niños(as) ${ }^{1}$.

Datos del Ministerio de Educación año 2016. Considera instituciones que solo ofrecen educación parvularia. No se contemplan establecimientos de educación en general que también ofrecen educación preescolar (Transición 1 y 2). 
La matrícula de la educación parvularia regular en establecimientos reconocidos por el Estado ${ }^{2}$ ha aumentado en los establecimientos particulares subvencionados, la que en 12 años se duplicó (de 100.000 a más de 200.000 estudiantes). Por el contrario, en establecimientos públicos la matrícula del nivel cayó casi un $10 \%$ (CHILE, 2017). Estas cifras son consistentes con la tendencia migratoria de estudiantes desde establecimientos públicos a privados subvencionados. Esta política fue instalada con la reforma conservadora de 1980, y no ha podido ser revertida en democracia, pese a las políticas impulsadas en este plano, tendencia que también se observa en la actualidad en forma creciente en algunos países de la región.

No obstante, para el año 2013, al considerar la educación parvularia en su conjunto, es al único nivel del sistema en que el financiamiento de recursos fiscales es mayor al promedio de la OCDE. En términos relativos, entre el 2003 y 2015 la cobertura educacional aumentó del $30 \%$ al $50 \%$ y más del doble en niños(as) de 3 años (23\% al 51\%), igual que en el caso de niños de 4 años, donde subió del $42 \%$ al 83\% (ORGANIZACIÓN PARA LA COOPERACIÓN Y EL DESARROLLO ECONÓMICO, 2017, p.107).

Entre el 2014 y 2017 se generaron 45 mil nuevos cupos en salas cunas y jardines infantiles públicos para niños(as) de 0 a 4 años. A estas vacantes se sumaron 25 mil para el 2018, representando un aumento del $31 \%$ de la oferta pública del nivel.

Pese a las positivas cifras de cobertura que muestra la educación parvularia en la última década, su participación al 2013 se mantiene bajo el promedio de la OCDE para niños(as) menores de 6 años, y especialmente para aquellos(as) de 2 y 3 años. Estos datos ocultan inequidades en el acceso a la educación parvularia, particularmente en la infancia "más temprana" (ORGANIZACIÓN PARA LA COOPERACIÓN Y EL DESARROLLO ECONÓMICO, 2017, p.108).

Diversos estudios confirman que en Chile las posibilidades de acceso a la educación parvularia son mayores en los quintiles más ricos de la población (ORGANIZACIÓN PARA LA COOPERACIÓN Y EL DESARROLLO ECONÓMICO, 2015) y en zonas urbanas más que rurales (BERTRAM; PASCAL, 2016). También son insuficientes los mecanismos del Estado para el acceso de grupos minoritarios. Por ejemplo, solo el $2,4 \%$ de niños(as) con necesidades especiales están matriculados(as) en jardines infantiles y salas cuna públicas. Por tal motivo, es fundamental desarrollar la infraestructura y equipamiento adecuados,

\footnotetext{
Sin incluir la matrícula de educación parvularia de JUNJI, Integra, salas cunas y jardines privados sin reconocimiento oficial del Estado.
} 
enfocándose en la población rural/urbana de bajos ingresos (BERTRAM; PASCAL, 2016). Para la OCDE, "la calidad y la cobertura de educación parvularia ha sido muy desigual en Chile" (ORGANIZACIÓN PARA LA COOPERACIÓN Y EL DESARROLLO ECONÓMICO, 2017, p. 80).

Complementariamente, el reciente y masivo fenómeno de migración extranjera genera desafíos para la educación parvularia. Los inmigrantes representan el 2\% de la población, aunque esta proporción está en franco aumento (INSTITUTO NACIONAL DE ESTADÍSTICAS, 2018). La formulación de la nueva versión de las Bases Curriculares de la Educación Parvularia refleja ese espíritu: “... destaca elementos que responden a nuevos requerimientos y énfasis de formación para la primera infancia, a saber: inclusión social, diversidad, interculturalidad, el enfoque de género, la formación ciudadana, el desarrollo sostenible, entre otros" (CHILE, 2018, p. 9)3.

Al tenor de esta evidencia, se requiere saber más de lo que ocurre en la gestión de los centros de educación parvularia, fenómeno prácticamente inexplorado (ORGANIZACIÓN PARA LA COOPERACIÓN Y EL DESARROLLO ECONÓMICO, 2017), razón por la cual el trabajo incursiona en su conocimiento, tratándose de un ámbito clave para Chile y otros países que han apostado a su fortalecimiento como estrategia de alto impacto sobre el sistema en su conjunto.

\section{Políticas Educativas y Modelo de Gestión del Centro Educativo Parvulario}

En el año 2013, prácticamente el $85 \%$ de niños y niñas de cuatro años y más del 90\% de los de cinco años, tenían matrícula en el sistema educativo (CHILE, 2017). "Este explosivo aumento de la cobertura se dio con mayor énfasis en los hogares más pobres, donde casi se ha duplicado" (ADLERSTEIN, 2012, p. 38). Además, durante esta década se inicia la reforma curricular del nivel, a saber: "Bases Curriculares de la Educación Parvularia", que estableció nuevos ámbitos de perfeccionamiento, orientaciones para proyectos de implementación curricular, material de apoyo, seguimiento y evaluación. El gobierno de entonces (2010-2014) continuó los progresos implementados, promulgándose la Ley de Aseguramiento de la Calidad (CHILE, 2011), la que instaló la discusión pública de una nueva arquitectura institucional para el sector.

3 Temas críticos en la política chilena, a modo de ejemplo, el Vo Congreso chileno de investigadores en educación se centrará en los temas sindicados (CIIE, agosto de 2019) 
El gobierno siguiente (2014-2017) fortaleció el diseño de las políticas y normativas legales para el financiamiento, quedando centralizadas en el Ministerio de Educación, en la nueva Subsecretaría de educación parvularia. La evaluación de los establecimientos es responsabilidad de la Agencia de la Calidad de la Educación y la fiscalización de los recursos reside en la nueva Intendencia de Educación Parvularia (CHILE, 2015). Esta institucionalidad se oficializa el 2015 , originando transformaciones de interés en un horizonte de corto, mediano y largo plazo. Ambos organismos comenzaron a cumplir funciones definidas en la Política de Aseguramiento de la Calidad de Jardines Infantiles y Salas Cunas (AGENCIA DE CALIDAD DE LA EDUCACIÓN, 2015).

En cuanto al financiamiento, la ley establece que para los establecimientos públicos ${ }^{4}$ el primer ciclo de la educación parvularia debe ser gratuito, prohibiendo todo tipo de cobro a la familia (matrícula, mensualidad, etc.) debiendo ser instituciones sin ánimo de enriquecimiento, focalizándose en los niños más vulnerables. Sin embargo, de los 4 a 6 años se acepta la provisión mixta de los recursos, permitiéndose instituciones con fines de lucro y el copago a las familias. El último gran desarrollo de la educación parvularia guarda relación con la revisión y actualización de las nuevas Bases Curriculares de la Educación Parvularia, aprobadas a inicios del 2018 donde se define el marco curricular nacional, alineando los objetivos de aprendizaje del nivel con los de la enseñanza básica o primaria.

De acuerdo con este documento, el funcionamiento de las instituciones educativas depende de un modelo de gestión integral "para manejar la complejidad de los procesos dentro de un establecimiento de educación preescolar, propone organizar dicha complejidad en ámbitos de la gestión, exigiendo en todos ellos cierto logro de objetivos y metas, en una cultura de procesos" (JUNTA NACIONAL DE JARDINES INFANTILES, 2005, p. 95). Bajo esta inspiración, a partir del 2013, comienza en la JUNJI el proceso de certificación de calidad, cuyo objetivo es implementar un Sistema de Aseguramiento de la Calidad de la Educación Parvularia, estandarizado para todos los jardines infantiles públicos y privados del país. Este Modelo ha sido probado en distintas etapas, lo que ha permitido actualizar conceptos, formatos y metodologías. Su diseño se inicia el año 2003 con una modalidad participativa con representantes de las comunidades educativas en la definición de estándares 5 .

\footnotetext{
4 Para este caso, se define como "público" de acuerdo a la fuente de los recursos financieros y su organización jurídica: Municipios, JUNJI o Integra.

5 La transformación permanente es intrínseca a este, cuyas bases están definidas por un mejoramiento continuo de los procesos y estándares escogidos.
} 
Entre el 2004 y 2005, se piloteó el Modelo en una muestra de establecimientos, definiendo el formato basal en 2005, sustentado en estándares definidos en colaboración con el Centro Nacional de la Productividad y Calidad (ChileCalidad). A partir del año 2007 se inició su implementación en los jardines infantiles. Los procesos de autoevaluación se replican el 2009 y 2011, incorporando a jardines administrados por terceros con financiamiento de la JUNJI, que adhieren voluntariamente a su implementación (JUNTA NACIONAL DE JARDINES INFANTILES, 2013b, p. 5). A partir del 2013, su implementación se enmarca en el proceso de Certificación de Calidad, en tanto se lleva a cabo el cuarto proceso de autoevaluación del modelo (JUNTA NACIONAL DE JARDINES INFANTILES, 2013b, p. 6).

El Modelo consideró seis áreas y diecinueve dimensiones. Las áreas son: (i) Liderazgo, (ii) Gestión de los Procesos Educativos, (iii) Participación y Compromiso de la Familia y la Comunidad, (iv) Protección y Cuidado, (iv) Gestión y Administración de Recursos Humanos y Financieros; y (vi) Resultados. Cada establecimiento define un Comité de Calidad y deben efectuar una autoevaluación siguiendo las orientaciones del modelo. Los comités son responsables de asegurar que la comunidad educativa reciba la capacitación para realizar la autoevaluación. Posteriormente, un equipo externo valida la autoevaluación, entregando un Informe de Resultados. Concluye el proceso con la clasificación del jardín infantil en uno de los cuatro niveles de calidad: excelente, avanzado, medio y básico.

Aquellos elementos de gestión deficientes son priorizados de modo a maximizar los recursos, incluso de los jardines infantiles que obtienen el máximo puntaje. Los elementos de gestión deficientes se sistematizan en un Plan de Mejoramiento. Esta fase del proceso de autoevaluación cierra su ciclo para dar comienzo a uno nuevo, incorporando los aspectos a mejorar.

\section{Consideraciones metodológicas}

Se trata de un estudio exploratorio-cualitativo, aplicado a una población de educadoras de párvulos que trabajan en centros dedicados exclusivamente a esta función en la región del Maule. Corresponde a una muestra intencional de quienes laboran en instituciones públicas, sea como directoras o educadoras de aula. En estos centros trabajan exclusivamente mujeres. Se utilizó un cuestionario de respuestas abiertas validado mediante juicio de expertos ${ }^{6}(3$

\footnotetext{
6 Los expertos correspondieron a supervisoras de las instituciones participantes en el estudio. Se utilizó el Índice de validez de contenido (IVC) de Lawshe (1975).
} 
casos), y posteriormente por una muestra de ex educadoras- directoras ( 6 casos) similar a quienes eran parte de la población.

La aplicación se realizó en una actividad a la que fueron invitadas (Julio 2017), evento apoyado por las entidades públicas responsables, cuya tarea inicial fue responder el cuestionario señalado. La población eran profesionales del sector público que se hubiesen desempeñado al menos 4 años en el nivel.

Las dimensiones analíticas consideradas se generaron a partir del Marco de Dirección de la JUNJI, incluyendo: infraestructura y equipamiento: recursos humanos, gestión pedagógica (de las educadoras, del currículo, de las estrategias de enseñanza), del liderazgo y la convivencia escolar, y de los resultados alcanzados.

Por tratarse de un estudio inicial, sus pretensiones son identificar temas que pudiesen ser investigados posteriormente; no tiene aspiraciones de representatividad de las temáticas indagadas.

El cuestionario se aplicó a 31 educadoras-directivas (en adelante directoras) que cumplen labores de dirección de un centro de educación de párvulos, y 103 educadoras de aula (en adelante educadoras).

La mayoría de las educadoras (53\%) declaran tener como máximo grado académico la Licenciatura. Prácticamente 1/6 (16\%) posee grado de Magíster y 2 educadoras declaran tener el grado de doctor(a).

Las directoras y educadoras tienen una trayectoria laboral relevante, según el caso, como educadora de aula y/o directora. En promedio, señalan 17 años como docente y casi 5 años de experiencia en el cargo de directoras, valores sustantivos para el estudio. Al clasificarlas, según su cargo en la comunidad escolar, más de la mitad (53\%) de los casos corresponden a educadoras de aula, un quinto de las participantes (20\%) a directoras y el saldo (27\%) en su mayoría a desempeño de otros cargos directivos: Coordinadoras, Jefas técnicas o Supervisoras.

El análisis se orienta a (i) identificar los mayores problemas que directoras y educadoras de párvulos detectan en sus comunidades educativas, para (ii) precisar los desafíos que requieren de soporte más explícito. Sobre la base de estos antecedentes se formulan propuestas de políticas para el mejoramiento de la gestión educativa. 


\section{Resultados}

\subsection{Dificultades y desafíos en recursos de operación y gestión curricular}

En el ámbito de los Recursos, estos están comprendidos "como la dotación de implementos y capacidades del establecimiento educacional (normas, personal, prácticas) para asegurar el desarrollo de los docentes y paradocentes; la organización, mantención y optimización del trabajo y soportes en función del Proyecto Educativo y de los resultados de aprendizaje de los estudiantes" (CHILE, 2013, p. 7). Corresponde a una dimensión clave donde Chile ha invertido en múltiples aspectos, esencialmente en recursos de aprendizaje para los estudiantes y preparación de los docentes.

Los principales problemas señalados por las directoras se refieren a insuficiencias de infraestructura. Un segundo aspecto se asocia con la disponibilidad y capacidad de los recursos humanos y financieros, cuyas respuesta textuales y dominantes son: "...falta de profesionales competentes", “...recursos inadecuados", "...bajas remuneraciones".

Un segundo nudo de dificultades del área Recursos es la falta de personal profesional y de reemplazo por licencias médicas, más la insuficiente capacitación del personal. Las opiniones dominantes son: “...falta de insumos básicos (incluyendo internet)", "gestión inadecuada de recursos financieros".

Un tercer factor aborda la insuficiente dotación de materiales de enseñanza en los tiempos requeridos, así como dificultades asociadas al personal de reemplazo, fallas en infraestructura o la importancia del desarrollo de habilidades. Esto se refleja en respuestas como: "falta de recursos para la compra de materiales de aula", "falta de un bus de acercamiento por la dispersión geográfica de los estudiantes".

En referencia a la opinión de las educadoras, estas se centran en dos componentes plenamente coincidentes: primero, respecto a materiales de enseñanza y personal de apoyo al aula ( $60 \%$ de las respuestas) y segundo, con relación a los derivados de la infraestructura impropia para las tareas que deben asumir (40\%).

En resumen, los problemas asociados a los Recursos son fundamentales para directoras y educadoras en la implementación de los proyectos educativos institucionales, siendo un pre-requisito de calidad de los mismos. Complementariamente, se vinculan a las dificultades para disponer de personal 
adecuado en cantidad y calidad, y finalmente del material didáctico. Por ello es importante comprender el rol de "soporte" al desarrollo educativo de estos aspectos, los cuales no pueden ser soslayados en los procesos de masificación, y al no ser implementados con la logística requerida pueden terminar incidiendo negativamente en los resultados.

El segundo componente analizado, la Gestión curricular, definido como "las prácticas del establecimiento educacional para asegurar la sustentabilidad del diseño, implementación y evaluación de su propuesta curricular" (CHILE, 2013, p. 5), se refiere directamente a cómo se gestiona el programa educativo en términos de horas de trabajo, asignación de educadoras, apoyo pedagógico, uso de los recursos didácticos, etc.

$\mathrm{Al}$ respecto, las directoras centran sus respuestas en falta de tiempo para el trabajo colaborativo. Igualmente expresan el débil intercambio de experiencias y prácticas pedagógicas entre ellas. A eso suman la escasa innovación en el trabajo pedagógico y la reiteración de prácticas tradicionales: "escaso intercambio de experiencias pedagógicas entre educadoras", “...se requieren instancias de análisis de prácticas pedagógicas","...implementar prácticas innovadoras”.

También hay tensión en la distribución del tiempo de trabajo de educadoras y asistentes, a saber: “...inadecuada distribución de trabajo administrativo y pedagógico", "baja valoración del tiempo dentro del aula".

Una cuarta dimensión considera un frágil enfoque del trabajo hacia el aprendizaje de niños(as) y la necesidad de avanzar hacia mayor consenso en los criterios de evaluación. Las directoras señalan: "Prácticas pedagógicas alejadas de su intencionalidad”, “...escaso tiempo para completar documentación pedagógica”, "priorizar el aprendizaje de niños y niñas", “...evaluaciones de acuerdo a un reglamento unificado de los establecimientos".

Complementariamente, la opinión de las directoras referidas a la Gestión Curricular se relaciona con las dificultades en la distribución del tiempo entre tareas pedagógicas y administrativas, la falta de colaboración (y tiempo) de educadoras para compartir experiencias y la reticencia a la innovación pedagógica. Se destaca la necesidad de centrar el proceso en los requerimientos formativos de niños y niñas, ello se refleja en: “...falta de tiempo para proyecto Tablet 7 "; “...falta de tiempo para gestionar actividades extra-programáticas".

7 Dirigido al uso de este instrumento para el aprendizaje de niños y niñas. 
Las respuestas de directoras y educadoras muestran núcleos temáticos coincidentes en materia de recursos, (i) lo clave es la saturación del tiempo laboral por diversas tareas que deben asumir, (ii) la no provisión oportuna de los recursos materiales y de trabajo en los tiempos pedagógicos (incluyendo infraestructura que es un tema complejo), y (iii) la necesidad de disponer de personal con las competencias profesionales requeridas.

En gestión curricular se subraya la necesidad de compaginar el principio de centralidad formativa de niños y niñas con el trabajo educativo y los recursos pedagógicos, incluyendo las competencias del equipo de educadoras. Hay una llamada de atención en el aseguramiento del cumplimiento de la calidad del Proyecto Educativo y de sus resultados de aprendizaje que alerta en las prácticas de los jardines infantiles para asegurar la sustentabilidad de sus propuestas curriculares.

\subsection{Dificultades y desafíos del liderazgo pedagógico, convivencia y apoyo a estudiantes}

En esta dimensión coexisten dos componentes relevantes: liderazgo pedagógico, comprendido como "la labor de movilizar e influenciar a otros para articular y lograr los objetivos y metas compartidas" (LEITHWOOD et al, 2006) y la Convivencia escolar y apoyo a los estudiantes, asumida como "las prácticas que lleva a cabo el establecimiento educacional para considerar las diferencias individuales y la convivencia de la comunidad educativa favoreciendo un ambiente propicio para el aprendizaje" (CHILE, 2013, p. 6).

Las dificultades asociadas al Liderazgo que mencionan las directoras se centran en la falta de organización y consolidación de equipos de trabajo, producto de la alta rotación de las educadoras. Algunas respuestas representativas son: "dificultades para consolidar equipo de educadoras"; “... organización de equipo de trabajo ante fuertes cambios"; “...compromiso profesional y laboral”; "incidencia de patrones obsoletos de trato y cuidado de los niños de técnicos y educadoras".

Otro aspecto son las relaciones interpersonales: "tensión en las relaciones interpersonales"; "el personal no tiene confianza con la dirección". Esto demuestra la complejidad de la organización educativa y el liderazgo, sobre el cual hay que mejorar las competencias de las directoras para fortalecer los equipos y el manejo de las relaciones interpersonales, fenómeno que incide en la cohesión y compromiso laboral. 
Se suma a ello la multiplicidad de labores que desarrollan las directoras, la exigencia de tareas administrativas en su labor diaria. Respuestas destacadas son: "ser directora y educadora a la vez”, “...hace falta más colaboración y respaldo del sostenedor".

En este ámbito, educadoras directoras coinciden al señalar las dificultades de organizar los equipos de trabajo en aula (56\%), que se desglosa en equipos estables y trabajo coordinado; y las tensiones en las relaciones interpersonales $(35 \%)$, donde son destacadas las dificultades de generar relaciones funcionales y objetivas en organizaciones tan lábiles.

Respecto a la convivencia escolar y apoyo al estudiante, las directoras manifiestan conflictos entre las educadoras que repercuten en el clima laboral, siendo las respuestas más representativas: “... lograr buena convivencia entre el equipo y comunidad educativa"; "malas relaciones entre funcionarias"; “...clima organizacional tenso al interior del grupo docente".

En segunda instancia, expresan que hay tensión en las relaciones entre el personal y la necesidad de un mayor involucramiento de las familias en el proceso de aprendizaje, con opiniones como: “...hay conflictos y problemas de comunicación con familias"; “...hacer entender a las familias que los jardines no son asistenciales"; "más compromiso de la familia".

Las directoras mencionan problemas sustantivos para ejercer su liderazgo derivados de: su formación inicial, la organización del trabajo educativo, las prácticas en este plano, la inadecuada infraestructura y equipamiento, y de las tensiones entre los equipos docentes. En segunda instancia, un signo de los tiempos es la insatisfacción de educadoras -en todo el sistema escolar- con el compromiso de la familia en el proceso educativo, una materia compleja también para la educación parvularia.

En este mismo ámbito -convivencia escolar y apoyo al estudiante-, las educadoras señalan las dificultades de falta de compromiso del personal y prácticas para favorecer la no-discriminación y la diversidad sociocultural de los estudiantes, que impliquen mejor convivencia y apoyo a los mismos (65\%). También las educadoras expresan que es importante mejorar sus prácticas para generar una mejor convivencia escolar (35\%), con rúbricas adecuadas a los objetivos/metas para niños y niñas, para asegurar mayor involucramiento de la familia en los aprendizajes de sus hijos. Para alcanzar esta meta, expresan las educadoras, se necesita perfeccionar los canales de información y comunicación con los 
actores de la comunidad educativa y recibir observaciones y sugerencias de su parte al respecto.

La convergencia de opiniones en esta sección muestra que la convivencia intra-establecimiento educativo es fundamental y se transforma en una materia de gestión que pone en tensión al liderazgo, aspecto que es recurrente en estos aspectos (ROSISTOLATO; PRADO; MARTINS, 2018). Hay una tarea importante que enfrentar con procesos formativos atingentes a todos quienes se desempeñan en este nivel, seguido de un trabajo en equipo que aborde aquellas temáticas recurrentes, que levante las prácticas periódicas e incorpore nuevas experiencias, situación que requiere de una condición estructural diferente de organización del trabajo educativo, con mayor estabilidad laboral, de relación con la familia y la comunidad, con un diseño de funcionamiento que atienda estas problemáticas.

\subsection{Dificultades y desafíos de la dimensión Resultados}

El ámbito de los Resultados, se entiende en lo fundamental como los logros de aprendizaje y formativos de niños y niñas, siendo la consecuencia final del proceso de la educación en cada nivel. Si bien se trató del área con menos menciones, tanto de directoras como educadoras, los aspectos planteados son relevantes, dando cuenta de la necesidad de emplear criterios comunes y más explícitos a nivel nacional, sobre cuáles son los logros de aprendizaje y formativos esperados para los párvulos.

Aunque las nuevas bases curriculares del nivel (2018) van en este sentido, es importante precisar los estándares, para evitar la escolarización temprana del nivel. Es decir, que sus objetivos no se centren en la enseñanza primaria. En este plano se emitieron opiniones como: “... nos falta precisión sobre los resultados esperados, con mayor especificidad"; "es importante contar con estándares definidos que contribuyan a la mejor enseñanza del nivel"; "se necesitan criterios consensuados y precisos para saber mejor dónde debemos llegar".

Aunque algunas directoras expresaron su satisfacción con los resultados alcanzado dado el contexto y condiciones en que trabajan, hecho en sí positivo, algunas se refirieron expresamente a la necesidad de articular en mejor forma los niveles del sistema educativo local: parvulario, básico y medio, para obtener mejores logros a nivel general.

En síntesis, las directoras tienen clara la necesidad de definir los parámetros de resultados a los que deben llegar para optimizar en mejor forma su tarea, 
vinculando además su hacer al de los otros niveles del sistema. También hay un reconocimiento de lo logrado, considerando los recursos y condiciones en las que trabajan.

Por su parte, las educadoras se refieren de manera exclusiva a la falta de claridad sobre los logros de aprendizaje que debiese alcanzar la educación parvularia, lo que se traduce en ausencia de "...datos del establecimiento educacional que muestran el nivel de logro de los niños y niñas en los distintos ciclos y/o sub-ciclos", “...en algunos casos se exige en el Kínder enseñanza de escritura y lectura en el nivel de educación parvularia, pese a que no deben obligatoriamente leer y escribir, más bien es iniciarles en el proceso lector", mostrando la importancia de avanzar en estas materias dada la relevancia temática y universalización que va adquiriendo la educación parvularia.

En síntesis, respecto de los resultados esperados en este nivel de enseñanza, es importante precisarles en mayor grado, dado que son fundamentales para gestionar el currículo. Si bien no es visto por educadoras y directoras como un área crítica, si es estratégica para dar un salto cualitativo en este nivel de enseñanza y en su aporte al sistema escolar.

Finalmente, y en el plano de las convergencias de las dimensiones analizadas, en referencia a los componentes del Modelo de gestión expuesto, se requiere un soporte más explícito de este último, en cuanto su metodología permita definir oportunidades sustentadas en los principios del "Mejoramiento Continuo", a saber: el esfuerzo creciente y constante como elemento que potencia la calidad de la gestión de un Jardín Infantil. Por tanto, una vez diagnosticadas las dificultades y fortalezas de gestión por medio del Modelo en comento, se posibilita la generación de soluciones y refuerzos. Consecuentemente, se pudo observar que mayoritariamente las dificultades estaban asociadas al área de recursos y gestión curricular (60\%); un 35\% al de liderazgo y convivencia escolar; y un $5 \%$ al de resultados, componentes muy cercanos a los definidos por el Modelo.

Bajo el Modelo analítico considerado, las problemáticas deberían ser tratadas en un Plan de Mejoramiento armonizado con el Proyecto Educativo del centro infantil, para reducir las brechas y organizar las acciones, siendo un instrumento que permite identificar fortalezas y debilidades y precisar oportunidades de mejora, al entregar información oportuna para la toma de decisiones del proceso educativo. (JUNTA NACIONAL DE JARDINES INFANTILES, 2013a). 


\section{Debate final}

La temática en análisis, la gestión de los centros de educación parvularia en Chile, ha sido insuficientemente estudiada en el país. Cuestión relevante dado que en forma insistente el Estado nacional, en particular en las últimas dos décadas, ha invertido sendos recursos en forma significativa en este nivel, impactando en mayor cobertura y nuevos centros educativos para párvulos como estrategia clave para el mejoramiento del sistema educativo nacional, política que también es impulsada por otros países de la región con objetivos similares; de allí la importancia de adentrase en un campo complejo como es la organización educativa (SLATER et al, 2018).

A las insignes razones que sustentan esta política, la realidad confirma que las oportunidades educativas de niños y niñas son desiguales, favoreciendo a los segmentos sociales de mayor poder económico, y postergando además los sectores más vulnerables -urbanos y rurales-. Este es un fenómeno que la política pública busca corregir, pero con estrategias que, siendo positivas, al tenor de los antecedentes descritos, requieren de un rediseño y ordenamiento para incrementar su eficacia (DONOSO-DIAZ, 2018).

Ciertamente, esta materia en estudio posee componentes técnicos y otros políticos-ideológicos que se insertan en las tendencias crecientes a la privatización (abierta o encubierta) de los sistemas públicos de enseñanza (BALL; YOUDELL, 2009), de las que Chile ha sido miembro destacado desde los años 80 pasados, y como tal hay que comprender su devenir en este marco ampliamente estudiado (ANDERSON, 2018), lo que no implica compartir su diseño.

La notable expansión de la cobertura territorial del nivel parvulario se ha hecho a partir de un importante pero claramente insuficiente incremento de recursos. Por ende, en un marco de sustentabilidad de esta política, es fundamental, siguiendo las opiniones de las participantes, sincronizar el equipamiento e infraestructura con los requerimientos de calidad de las oportunidades educativas.

En este plano, la articulación vertical del sistema parvulario con la enseñanza escolar inmediatamente superior (básica) es relevante, como también lo es mejorar la organización y funcionamiento de los centros de párvulos, unificando sus procesos formativos pues hay diferencias importantes que, finalmente, terminan gravando a la población más pobre. Por lo mismo, la cobertura debe considerar no solamente asistencia al centro educativo sino equidad de procesos formativos, lo que corresponde a intervenir en la gestión curricular. 
En esta misma dimensión, se reconocen y fortalecen los objetivos propios del nivel parvulario, no solo aquellos funcionales a la continuidad del niño(a) en el sistema escolar. Ello requiere de un soporte sólido en la formación profesional de educadoras y asistentes de párvulos en los enfoques y técnicas actualizados, abordando la deficiente organización del trabajo educativo, debido a la falta de recursos pedagógicos y de competencias del equipo de educadoras en algunas dimensiones, como también a la importancia de actualizar la visión pedagógica que les sustenta.

En este marco, el fortalecimiento del liderazgo pedagógico no solo es una tarea de las directoras, sino de sus equipos directivos y de las orientaciones de gestión que deben reforzarse con estándares más definidos acerca de su desempeño, y de los resultados esperados de su trabajo en términos del impacto en los aprendizaje y formación de niños y niñas. Estas debilidades están asociadas a la alta rotación del personal profesional y del manejo -no siempre adecuado- de las relaciones interpersonales que impactan en el compromiso de sus integrantes. Los problemas de convivencia educativa, factor crítico en toda organización educativa, requieren de una estructura que les canalice y de formación adecuada y permanente de sus integrantes, a fin de instalar prácticas que favorezcan la diversidad sociocultural de los estudiantes, que conlleven a una mejor convivencia y de apoyo a los mismos.

Los resultados consignados muestran que el sistema ha sido capaz, con el esfuerzo de sus profesionales, de absorber una fuerte demanda de cobertura, pero requiere preocuparse por materias esenciales como las señaladas, para que estas medidas sean sustentables en el tiempo y configuren una política pública efectiva de incorporación de la población más vulnerable a la educación inicial, como estrategia potente del mejoramiento educativo.

En atención a lo anterior, se advierten desafíos relevantes para dar cuenta de la complejidad descrita. Instalar procesos de mejoramiento de la gestión de los centros educativos de párvulos es una tarea necesaria que implica adecuar sus procesos, reevaluar las herramientas en este plano y desarrollar las competencias requeridas en sus equipos directivos, varias de las cuales se han esbozado.

En términos más específicos, es relevante atender los siguientes aspectos:

(i) La educación parvularia comparte problemas de calidad, algunos de los cuales radican en que no existe consenso sobre los alcances de los estándares de excelencia del sector. Si bien hay avances institucionales al respecto, en particular 
el Sistema de Aseguramiento de la Calidad, estos debieran centrarse en definir criterios de calidad contextuales y consensuados a nivel de comunidad escolar, que sean pertinentes a los diversos contextos formativos.

(ii) Las competencias de directoras y educadoras muestran brechas de eficacia que deben cerrarse. Por tanto, junto a aspectos básicos de estabilidad y permanencia del personal profesional que deben ser resueltos, su formación debe ser atendida por medio de una política pública nacional sostenible, independiente del gobierno de turno.

(iii) La insatisfacción de educadoras y directoras respecto de la falta de compromiso de la familia en el proceso educativo, dimensión compleja y presente en todos los niveles del sistema educativo, dada la particularidad que adquiere por la mayor cercanía apoderado-párvulos en este sector, debe ser potenciada como ventaja comparativa para desarrollar buenas prácticas para posteriormente extrapolarlas a otros niveles.

(iv) Frente a la necesidad de atender la convivencia de todos los actores intraestablecimiento educativo, se necesita instalar prácticas de liderazgo pedagógico efectivas que fortalezcan el trabajo de equipo, no sin antes asociarlas a condiciones laborales de mayor calidad.

(v) En materia de resultados, si bien se reconocen los aportes de las nuevas bases curriculares en la educación parvularia (2018), hay objetivos propios del nivel que hay que salvaguardar para reducir la escolarización temprana, necesitándose estándares propios de mayor calidad para los subniveles respectivos.

Finalmente, respecto a las propuestas de políticas para el mejoramiento de la gestión educativa de la educación parvularia, es importante que se resuelvan ciertos aspectos:

(i) Contar con herramientas de gestión y pedagógicas para hacer más efectiva su labor directiva, requiriéndose cambios sustantivos en la formación inicial y continua que estimulen la capacitación, con una institucionalidad que guíe y respalde la formación y mejoramiento continuo.

(ii) Promover, por parte del Estado, el Marco para la Buena Enseñanza de Educación Parvularia, que nace de su equivalente para el ámbito escolar, el cual está en su fase final de validación (marzo de 2019). Este marco incluye 
orientaciones pedagógicas y prácticas, considerando procesos de capacitación, acompañamiento, monitoreo y evaluación de sus educadoras.

(iii) Finalmente, fomentar, por parte del Estado, una mayor participación de los actores de este nivel educativo, consensuando metas que signifiquen un mejoramiento sostenido y acorde a los estándares definidos, con estrategias claras de difusión para generar transferencia de conocimientos y continuidad. 


\title{
The management of preschool centers in the Maule region (Chile): visions from educational practice
}

\begin{abstract}
For more than two decades, Chile has promoted important initiatives of greater coverage and quality of early childhood education, which require - for their sustainability - to reinforce the management processes of the educational centers, a problem that does not have systematic antecedents. In response to this situation, we seek to identify the obstacles that preschool centers directors and educators consider to be the most important, in order to propose policies for their improvement. In this context, this study (exploratoryqualitative) was developed, based on an open response questionnaire. The results identify key problems that require prompt attention, in order to contribute to the greater impact of the indicated initiatives.
\end{abstract}

Keywords: Preschool education. Educational management. Educational policy. Sustainability. Chile

\section{A gestão de centros de educação infantil na região do Maule (Chile): visões da prática educacional}

\section{Resumo}

Há mais de duas décadas, o Chile promove importantes iniciativas de maior abrangência e qualidade da educação infantil, que exigem - para sua sustentabilidade - reforçar os processos de gestão dos centros educacionais, problema que não tem antecedentes sistemáticos. Em resposta a essa situação, buscamos identificar os obstáculos que diretores e educadores de escolas de berçário consideram como os mais importantes, a fim de propor politicas para sua melhoria. Nesse contexto, o estudo foi desenvolvido (exploratório-qualitativo), a partir de um questionário de resposta aberta. Os resultados identificam os principais problemas que requerem atenção imediata, a fim de contribuir para o maior impacto das iniciativas indicadas.

Palavras-chave: Educação infantil. Gestão educacional. Política educacional. Sustentabilidade. Chile 


\section{REFERENCIAS}

ADLERSTEIN, C. La política pública de la educación parvularia chilena: una mirada desde la historia y su actualidad. Revista Docencia, Santiago, v. 17, n. 48 , p. $30-45$, dic. 2012.

\section{AGENCIA DE CALIDAD DE LA EDUCACIÓN - ACE.}

Estudio calidad educativa en educación parvularia: experiencias internacionales y representaciones sociales nacionales, informe final. Santiago, 2015. Disponible en: $<$ http://archivos. agenciaeducacion.cl/Informe_Estudio_Calidad_Educacion_Parvularia_2015. pdf $>$. Acceso en: 18 oct. 2018.

ANDERSON, J. E. Entrevista líderes educativos. Valparaíso: Centro de Liderazgo para la Mejora Escolar, 2018. Disponible en: <http://lideres educativos.cl>. Acceso en: 15 abr. 2018.

BALL. S.; YOUDELL. D. Privatización encubierta en educación pública. Londres: Universidad de Londres, 2009.

BERTRAM, T.; PASCAL, C. Early childhood policies and systems in eight countries: findings from IEA's early childhood education study. Hamburg: International Association for the Evaluation of Educational Achievement, 2016.

BLANCO, R. Educación inclusiva y atención a la diversidad. En: PERALTA, V.; HERNÁNDEZ, L. (Coords.). Antología de experiencias de la educación inicial iberoamericana. Madrid: Organización de Estados Iberoamericanos, 2012. p. 80-6.

CHILE. Ley N $\mathrm{N}^{\mathrm{0}} 20.529$, de 11 de agosto de 2011. Sistema nacional de aseguramiento de la calidad de la educación parvularia, básica y media. Boletín Legislativo, 27 ago. 2011.

. Ley $\mathrm{N}^{\mathrm{o}} 20.835$, de 28 de abril de 2015. Crea la subsecretaría de educación parvularia, la intendencia de educación parvularia. Boletín Legislativo, 5 mayo 2015.

Ministerio de Educación. Bases curriculares de la educación parvularia. Santiago, 2001a. Disponible en: $<$ http://parvularia.mineduc.cl/ wp-content/uploads/sites/34/2016/05/201308281105060.bases_curriculares_ educacion_parvularia.pdf $>$. Acceso en: 12 oct. 2018. 
CHILE. Ministerio de Educación. La educación parvularia en Chile. Santiago: Unidad de Educación Parvularia, 2001b. (Serie Educación Parvularia 2001: aportes a la reflexión y a la acción. 1.7.). Disponible en: $<$ http://ww2. educarchile.cl/UserFiles/P0001\%5CFile\%5Ceduc_parvularia_en_chile.pdf $>$. Acceso en: 16 oct. 2018.

. Ministerio de Educación. Modelo de calidad de la gestión escolar. Santiago, 2013. Disponible en: <http://www.agenciaeducacion.cl/ wp-content/uploads/2013/02/Modelo-de-Calidad-del-Gestión-Escolar.pdf $>$. Acceso en: 02 oct. 2018.

. Ministerio de Educación. Hoja de ruta: definiciones de política para una educación parvularia de calidad. Santiago: Subsecretaría de Educación Parvularia, 2016. Disponible en: $<$ https://parvularia.mineduc.cl/wpcontent/uploads/sites/34/2017/02/HOJA-DE-RUTA-PDF-VERSIÓN-2017. pdf>. Acceso en: 01 oct. 2018.

. Ministerio de Educación. Estadísticas de la educación 2016. Santiago, 2017. Disponible en: $<$ https://centroestudios.mineduc.cl/wp-content/ uploads/sites/100/2017/07/Anuario_2016.pdf>. Acceso en 20 oct. 2018.

. Ministerio de Educación. Bases curriculares educación parvularia. Santiago, 2018. Disponible en: $<$ https://parvularia.mineduc.cl/wp-content/ uploads/sites/34/2018/03/Bases_Curriculares_Ed_Parvularia_2018.pdf $>$. Acceso en: 10 agosto 2018.

DONOSO-DIAZ, S. La nueva institucionalidad subnacional de educación pública chilena y los desafíos de gestión para el sistema escolar. Educar em Revista, Curitiba, v. 34, n. 68, p. 29-48. mar./abr. 2018. https://doi. org/10.1590/0104-4060.57480

HERRERA, M. O.; BELLEI, C. (Eds.). ¿Qué se sabe sobre la calidad de la educación parvularia chilena? Santiago: Unicef, 2002. (Ciclo de debates: desafíos de la política educacional).

INSTITUTO NACIONAL DE ESTADÍSTICAS - INE. Censo abreviado de población y vivienda. Santiago, 2018.

JUNTA NACIONAL DE JARDINES INFANTILES - Junji. Hacia un sistema de acreditación de calidad: salas cuna y jardines infantiles. Santiago: Maval, 2005. 
JUNTA NACIONAL DE JARDINES INFANTILES - Junji. Manual del modelo de gestión de calidad de la educación parvularia. Santiago, 2013a. Disponible en: < http://www.modelodecalidad.junji.gob.cl/documentos/ Manual\%20del\%20MGCEP.pdf>. Acceso en: 4 oct. 2018.

- Guía de autoevaluación: modelo de gestión de calidad de la educación parvularia, jardines infantiles clásicos, vía transferencia de fondos y particulares. Santiago, 2013b. Disponible en: $<$ http://www.modelodecalidad. junji.gob.cl/documentos/Gu\%C3\%ADa\%20de\%20Autoevaluación $\% 20 J . I \% 20$ Clásicos,\%20VTF\%20y\%20Particulares.pdf>. Acceso en 20 oct. 2018.

LAWSHE, C. H. A quantitative approach to content validity. Personnel Psychology, v. 28, n. 4, p. 563-75, dic. 1975. https://doi.org/10.1111/j.1744-6570.1975.tb01393.x

LEITHWOOD, D. et al. Successful school leadership: what it is and how it influences pupil learning. London: University of Nottingham, 2006.

ORGANIZACIÓN PARA LA COOPERACIÓN Y EL DESARROLLO ECONÓMICO - OCDE. The ABC of gender equality in education: aptitude, behaviour, confidence. Paris, 2015.

. Educación en Chile. Santiago: Ministerio de Educación, 2017.

ROSISTOLATO. R.; PRADO, A. P.; MARTINS, L. R. La realidad de la escuela y la recepción de las políticas educacionales. Ensaio: Avaliação e Políticas Públicas em Educação, Rio de Janeiro, v. 26, n. 98, p. 112-32, ene./ mar. 2018. https://doi.org/10.1590/s0104-40362018002601074

SLATER, C. et al. Liderazgo en los directivos principales: hallazgos de un estudio internacional comparado. En: WEINSTEIN, J.; MUÑOZ, G. (Eds.). Cómo cultivar el liderazgo educativo: trece miradas. Santiago: Centro de Desarrollo de Liderazgo Educativo, 2018. p. 299 -322 


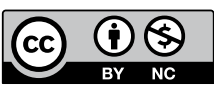

\section{Informações dos autores}

Nibaldo Benavides-Moreno: Profesor de Enseñanza Media, Doctor en Educación, Profesor Investigador del Instituto de Investigación y Desarrollo Educacional, Universidad de Talca. Contato: nbenavides@utalca.cl

iD http://orcid.org/0000-0003-4482-7205

Sebastián Donoso-Díaz: Educador, Doctor en Educación, Profesor Investigador del Instituto de Investigación y Desarrollo Educacional, Universidad de Talca. Contato: sdonoso@utalca.cl

iD https://orcid.org/0000-0002-4744-531X

Daniel Reyes Araya: Economista. Magister en Gerencia y Gestión Pública, Profesor del Instituto de Investigación y Desarrollo Educacional, Universidad de Talca. Contato: dreyes@utalca.cl

(iD) https://orcid.org/0000-0003-0593-8033

Tomas Neira Iturrieta: Economista, Magíster en Políticas Públicas. Investigador del Centro de Estudios del Ministerio de Educación de Chile. Contato: tneira@gmail.com

iD https://orcid.org/0000-0002-2130-8691 